\title{
Super-stable homogeneous iodinated formulation technology for improving the therapeutic effect of patients with advanced hepatocellular carcinoma
}

\author{
Pan He ${ }^{1,2 \#}$, Furui Zhong ${ }^{1 \#}$, Bin Luo ${ }^{1}$, Guosong Luo ${ }^{3}$, Xuewen Wang ${ }^{3}$, Xianming Xia ${ }^{1,2}$, Bo Li ${ }^{1,2}$ \\ ${ }^{1}$ Department of General Surgery (Hepatobiliary Surgery), The Affiliated Hospital of Southwest Medical University, Luzhou, China; ${ }^{2}$ Academician \\ (Expert) Workstation of Sichuan Province, Luzhou, China; ${ }^{3}$ Department of Hepatobiliary surgery, Zigong Fourth People's Hospital, Zigong, China
}

\#These authors contributed equally to this work.

Correspondence to: Bo Li. Department of General Surgery (Hepatobiliary Surgery), The Affiliated Hospital of Southwest Medical University, Luzhou, China. Email: liboer2002@126.com.

Provenance and Peer Review: This article was a free submission to the journal. The article did not undergo external peer review.

Comment on: Chen H, Cheng H, Dai Q, Cheng Y, Zhang Y, Li D, Sun Y, Mao J, Ren K, Chu C, Liu G. A superstable homogeneous lipiodol-ICG formulation for locoregional hepatocellular carcinoma treatment. J Control Release 2020;323:635-43.

Submitted Jun 08, 2020. Accepted for publication Aug 13, 2020.

doi: 10.21037 /qims-20-741

View this article at: http://dx.doi.org/10.21037/qims-20-741

Liver cancer is one of the most common malignant tumors in China. Worldwide, the incidence of primary liver cancer is about $15.3 / 100,000$ and the mortality rate is about $14.3 / 100,000$. Among male patients who died of cancer in 2018, liver cancer was the second-leading cause of cancer death (1). At present, surgical resection of hepatocellular carcinoma (HCC), also referred to as partial hepatectomy, is still the first choice for the treatment of HCC (2). However, due to the growth characteristics of HCC, there are no obvious symptoms in the early stages. When symptoms, such as fever, liver pain, systemic inflammation and abdominal swelling appear, the disease has mostly developed to the middle and late stages, the tumor diameter is large, the rate of radical resection is low, and prognosis is poor. Massive liver tumors usually have a diameter of $\geq 10 \mathrm{~cm}$, abundant blood supply, and tend to compress and invade the edge of the first, second or third hepatic hilum. Accordingly, their surgical removal involves a high degree of difficulty, large trauma, and more bleeding. They are often accompanied by satellite foci and intrahepatic microscopic cancer, with low surgical cure rate, which contribute to their postoperative relapse (3). Indeed, it has been reported that after the radical resection the 5 -year survival rate of patients with massive liver cancer tumors has been only $12.2 \%$ to $29.3 \%$ (4). Therefore, how to downstage a massive HCC to improve the rate of surgical resection, and how to reduce the trauma during resection and the rate of postoperative recurrence are important research questions to improve the prognosis of patients with advanced HCC.

Besides their large size and abundant blood supply, massive liver tumors have complex relationship with the surrounding structure with small residual liver volume after surgical removal (3). The surgical resection rate and postoperative effect can be improved by reducing preoperative tumor volume and reducing or blocking nourishing blood vessels around tumors so to increase disease-free liver volume. At present, commonly used methods include conventional transcatheter arterial embolization (TAE) and transcatheter arterial chemoembolization (TACE) (5). The second approach is to carefully evaluate the liver tumor and liver reserve function before surgery in order to select the appropriate 
surgical section. The third approach is to accurately locate the primary liver tumor, satellite foci and intrahepatic metastases, and the resection margin of the liver tumor during the surgical procedure to ensure the complete resection of the tumor, improve the complete resection rate of small lesions, and reduce the postoperative recurrence rate. Preoperative adjuvant TAE/TACE can be used to determine the size and blood supply of the tumor, embolize the blood supply artery of the tumor, necrotize the tumor, reduce the volume, which can reduce the tumor stage, increase the rate of surgical resection, reduce resection trauma and blood loss, and provide a way to reduce the risk of surgery $(6,7)$. However, it has been reported that the preoperative adjuvant TAE/TACE procedure may delay the optimal time for surgery and increase the chance of tumor metastasis. Therefore, a new clinical diagnosis and treatment technique is needed to overcome the shortcomings of TAE/ TAC-assisted HCC resection, to improve tumor resection rate and ensure complete tumor resection while avoiding small intraoperative liver cancer lesions and residual cancerous tissue. To improve the short-term treatment effect and long-term survival rate of patients, surgical trauma and blood loss during resection should be reduced, and the remaining normal liver volume should be maximized while reducing the postoperative recurrence rate.

Indocyanine green (ICG) is a fluorescent reagent used in clinical detection of liver function. It emits nearinfrared light at about $800 \mathrm{~nm}$ when excited by light of wavelength in the range of 750-810 nm, which can provide navigation capability for surgical resection with the aid of a fluorescence imaging system $(8,9)$. At the same time, the preoperative TAE-assisted technique is combined with intraoperative fluorescence molecular imaging to compensate for each other's deficiency. This combined technique is expected to provide a way to evaluate the preoperative liver reserve function, and improve the resection rate of massive HCC, as well as the short-term and long-term therapeutic effect of resection. However, since ICG is a small molecule, if ICG is injected intravenously before tumor embolization, the stability of the injected ICG is limited, and it is easily metabolized and removed quickly, and the fluorescence performance is reduced due to agglomeration quenching (10). If ICG is administered intravenously after tumor embolization, the absorption function of liver cancer cells is further impaired due to TAE/TACE embolization of the blood supply artery of the tumor and tumor necrosis, thus preventing the infiltration and distribution of ICG in the tumor tissue. For this reason, the surgeons usually use TAE to manually mix the ICG and iodized oil to prepare the emulsion in advance for surgical navigation after embolization. Although this method is simple and easy to implement, the resulting hand-adjusted emulsion is extremely unstable and will delaminate after standing for a while. After the emulsion is injected into the lesion through the artery, the precipitated ICG will be metabolized and cleared before surgery, resulting in poor surgical navigation effect. Recently, Liu's team at Xiamen University, China, developed a super-stable homogeneous iodinated formulation technology (SHIFT) to overcome the difficulties faced in HCC treatment. This technique skillfully combines the embolization agent iodide oil with the fluorescence agent ICG to prepare green, safe, stable, uniform, practical and efficient iodol-ICG reagents (referred to as SHIFTs), which have been shown to have potential clinical value as suggested by in vivo and in vitro experimental results (Figure 1) in a study recently published in the Fournal of Controlled Release (11).

SHIFT uses a physical method to achieve the complete dispersion of the solute in the solvent without adding any additional chemical adjuvant, such as activators, cosolvents and dispersants. SHIFTs prepared by this method are composed of iodine oil and ICG for clinical use, which ensure the safety and verify the effectiveness of the drug in vivo. When left at room temperature for 30 days during an in vitro study, SHIFTs still maintain a good dispersion state without precipitation. In addition, the fluorescence, photothermal and photoacoustic properties of ICG in SHIFTs made by this technology are all improved to a certain extent and play a protective role. Particularly in terms of the fluorescence performance of ICG, which due to the stability of iodized oil after liver cancer embolism, this method not only prevent the quenching of its fluorescence, but also extend the fluorescence lifetime of ICG (11). This feature provides a longer time window for the implementation of intraoperative fluorescent navigation in liver cancer resection after downstaging the liver cancer tumor, and is anticipated to further improve the chance of resection of large and advanced liver cancer. In addition, the SHIFTs adopt the TAE interventional administration method, which has an embolization effect equivalent to that of lipiodol. Due to the embolization of the blood supply artery of the tumor, the tumor is necrotic, its volume is reduced, which not only contributes to reduce the extent of surgical trauma and bleeding, but also reduces the risk 


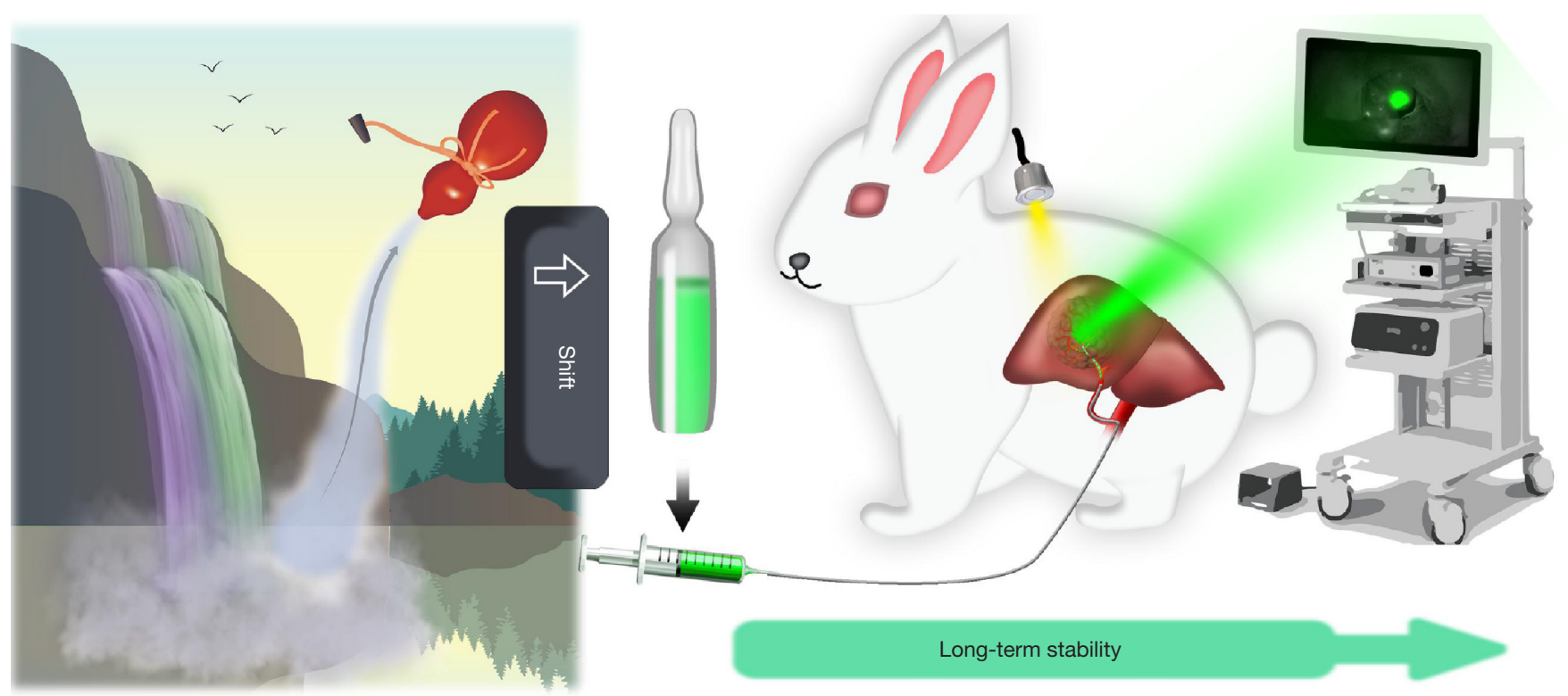

Figure 1 A schematic diagram showing the prepared super-stable homogeneous iodinated formulation technology (SHIFT) for transcatheter arterial embolization. The lipiodol (purple flow) and indocyanine green (ICG, green flow) are introduced into a reactor (lake), and the iodol-ICG SHIFTs (gourd) with long-term stability can act as an embolic agent and tumor bulb for imaging-guided hepatocellular carcinoma resection (11). Figures adopted with permission from (11).

of surgery. Additionally, it allows the surgeon to clearly and accurately identify the primary tumor and metastatic lesions, as well as to distinguish the boundary between the cancer tissue and normal tissue to prevent the recurrence and metastasis of the lesion and maximize the retention of normal liver tissue so as to ensure the recovery of liver function (12). In addition, the SHIFTs have better fluorescence navigation performance than the free ICG solution. This feature can better guide the resection of liver cancer after the reduction of mid-advanced huge liver cancer, which is of great importance to avoid the residual tumor tissue and reduce the rate of postoperative recurrence and reoperation.

Furthermore, through the extended analysis of SHIFT technology, it has been suggested that it can have broad application prospects in the medical field (13). The TACE therapy of liver cancer also involves the infusion of chemotherapy drugs into the embolization agent and their injection into the tumor through the artery, but the instability of the drug in the embolization agent due to the sudden release of the drug will cause acute side effects (14). It is believed that not only ICG but also other chemotherapeutic molecules can be processed through the SHIFT technology to prepare uniformly dispersed lipiodol- chemotherapy preparations. Thus, TACE for liver cancer will largely alleviate the adverse effects caused by the sudden release of the drug and improve its therapeutic effect. Moreover, if chemotherapeutic drugs are added on the basis of SHIFTs, it is expected that they can embolize the tumor blood vessels after entering the blood vessels, and cause the chemotherapeutic drugs to act inside the tumor for a longer time, which will greatly reduce tumor metastasis and lead to a more thorough tumor necrosis, which ultimately leads to the effect of the reduced tumor stage more substantial. On the other hand, for patients with successful downstaging, the fluorescent navigation ability will not be lost during liver cancer resection. In fact, for patients with unsuccessful downstaging, their treatment will not be delayed, but the effect of embolization will be improved.

In summary, the fluorescence-guided tumor resection after SHIFTs tumor downstaging opens the door for future clinical applications and has important practical significance to further improve the overall levels of quality treatment for liver tumors.

\section{Acknowledgments}

Funding: None. 


\section{Footnote}

Conflicts of Interest: All authors have completed the ICMJE uniform disclosure form (available at http://dx.doi. org/10.21037/qims-20-741). The authors have no conflicts of interest to declare.

Open Access Statement: This is an Open Access article distributed in accordance with the Creative Commons Attribution-NonCommercial-NoDerivs 4.0 International License (CC BY-NC-ND 4.0), which permits the noncommercial replication and distribution of the article with the strict proviso that no changes or edits are made and the original work is properly cited (including links to both the formal publication through the relevant DOI and the license). See: https://creativecommons.org/licenses/by-nc-nd/4.0/.

\section{References}

1. Yang JD, Roberts LR. Epidemiology and management of hepatocellular carcinoma. Infect Dis Clin North Am 2010;24:899-919, viii.

2. Song TJ, Ip EW, Fong Y. Hepatocellular carcinoma: current surgical management. Gastroenterology 2004;127:S248-60.

3. Loffroy R, Favelier S, Chevallier O, Estivalet L, Genson PY, Pottecher P, Gehin S, Krausé D, Cercueil JP. Preoperative portal vein embolization in liver cancer: indications, techniques and outcomes. Quant Imaging Med Surg 2015;5:730-9.

4. Liau KH, Ruo L, Shia J, Padela A, Gonen M, Jarnagin WR, Fong Y, D'Angelica MI, Blumgart LH, DeMatteo RP. Outcome of partial hepatectomy for large $(>10 \mathrm{~cm})$ hepatocellular carcinoma. Cancer 2005;104:1948-55.

5. Wang YX, Zhu XM, Liang Q, Cheng CH, Wang W, Leung $\mathrm{KC}$. In vivo chemoembolization and magnetic resonance imaging of liver tumors by using iron oxide nanoshell/doxorubicin/poly (vinyl alcohol) hybrid composites. Angew Chem Int Ed Engl 2014;53:4812-5.

6. Sasaki A, Iwashita Y, Shibata K, Ohta M, Kitano S, Mori

Cite this article as: He P, Zhong F, Luo B, Luo G, Wang X, Xia X, Li B. Super-stable homogeneous iodinated formulation technology for improving the therapeutic effect of patients with advanced hepatocellular carcinoma. Quant Imaging Med Surg 2020;10(11):2223-2226. doi: 10.21037/qims-20-741
M. Preoperative transcatheter arterial chemoembolization reduces long-term survival rate after hepatic resection for resectable hepatocellular carcinoma. Eur J Surg Oncol 2006;32:773-9.

7. Forner A, Llovet J M, Bruix J. Chemoembolization for intermediate HCC: is there proof of survival benefit? J Hepatol 2012;56:984-6.

8. Zhao M, Dong L, Liu Z, Yang S, Wu W, Lin J. In vivo fluorescence imaging of hepatocellular carcinoma using a novel GPC3-specific aptamer probe. Quant Imaging Med Surg 2018;8:151-60.

9. Lee SS, Kim H, Sohn DK, Eom JB, Seo YS, Yoon HM, Choi Y. Indocyanine green-loaded injectable alginate hydrogel as a marker for precision cancer surgery. Quant Imaging Med Surg 2020;10:779-88.

10. Lajunen T, Nurmi R, Wilbie D, Ruoslahti T, Johansson NG, Korhonen O, Rog T, Bunker A, Ruponen M, Urtti

A. The effect of light sensitizer localization on the stability of indocyanine green liposomes. J Control Release 2018;284:213-23.

11. Chen H, Cheng H, Dai Q, Cheng Y, Zhang Y, Li D, Sun Y, Mao J, Ren K, Chu C, Liu G. A superstable homogeneous lipiodol-ICG formulation for locoregional hepatocellular carcinoma treatment. J Control Release 2020;323:635-43.

12. Terasawa $M$, Ishizawa $T$, Mise $Y$, Inoue $Y$, Ito $H$, Takahashi Y, Saiura A. Applications of fusion-fluorescence imaging using indocyanine green in laparoscopic hepatectomy. Surg Endosc 2017;31:5111-8.

13. Chen H, Cheng H, Wu W, Li DF, Mao JS, Chu CC, Liu G. The blooming intersection of transcatheter hepatic artery chemoembolization and nanomedicine. Chin Chem Lett 2020;31:1375-81.

14. Lilienberg E, Dubbelboer IR, Karalli A, Axelsson R, Brismar TB, Ebeling Barbier C, Norén A, Duraj F, Hedeland M, Bondesson U, Sjögren E, Stål P, Nyman R, Lennernäs H. In Vivo Drug Delivery Performance of Lipiodol-Based Emulsion or Drug-Eluting Beads in Patients with Hepatocellular Carcinoma. Mol Pharm 2017;14:448-58. 